\title{
Experimental approach to eliminate Start/Stop defects in laser cladding
}

\author{
V. Ocelík, M. Eekma, I. Hemmati, M. Dutka \\ \& J. Th. M. De Hosson \\ Materials Innovation Institute M2i, Department of Applied Physics, \\ University of Groningen, The Netherlands
}

\begin{abstract}
Laser cladding represents an advanced hard facing technology for the deposition of a hard and corrosion resistant layer of controlled thickness onto a selected area of metallic substrate. When a circular geometry is required, the beginning and the end of the laser track coincide. This could lead to the formation of irregular microstructural features resulting in non-homogeneous properties. In this work a few different experimental strategies are examined to find a suitable approach in order to reduce this problem in the Start/Stop area. Laser cladding experiments were performed using $3.3 \mathrm{~kW}$ IPG Fiber laser, $40 \mathrm{~mm}$ in diameter SS304 steel substrate bar and two powders: Iron and Cobalt based. Standard metallographic methods, Scanning Electron Microscopy equipped with EDS and microhardness measurements were used to study the microstructure and properties inside the laser track overlapping areas created by different cladding strategies. These strategies were finally ranked according a few qualitative criteria and approaches are found which are beneficial over a situation where no special care is taken for the beginning and the end of laser clad track.
\end{abstract}

Keywords: laser cladding, microstructure, defects.

\section{Introduction}

Laser cladding is an advanced technique to deposit a thick coating onto a substrate in order to create a surface layer with specific properties suitable for certain applications. A high power laser beam is providing the heat source to melt simultaneously the substrate and the additional material, which will make up the coating, together. There are three different ways in which this additional 
material can be delivered. One of them is the powder injection directly into the laser beam and the melt pool created at the surface. In this method a coaxial [1] or a side powder [2] nozzle is used to blow the powder with the aid of a carrier gas. This method does not experience drawbacks compared to others (pre placed powder, wire feeding) where some problems with cladding on a complicated surface shape or with the process control are reported [3].

The most effective use of advantages of laser cladding technique is in the local surface improvement or in reparation of heavily damaged surfaces. Sometimes the laser cladding on the edges of circular openings [4] or flanges [5] is required. In this case not only the side overlapping of individual laser tracks (usually utilized to cover areas wider than single laser beam spot size) is required, but also the start of the laser track created at the beginning of the laser cladding process has to be overbuilt by the laser track end formed at the tail of the laser cladding process. While the side overlapping of individual laser tracks is widely studied in literature not only for laser cladding but also for laser hardening and laser melt injection processes, the Start/Stop overlapping and potential defects formed during the laser cladding in this area are not reported in the literature. It is possible that in many laser surface engineering laboratories they have their own approach how to treat this area and avoid potential defects.

However, the main aim of this study was to evaluate a few experimental approaches to eliminate geometrical and microstructural defects formed inside the Start/Stop zone. These approaches are simultaneously studied on two typical laser cladding coatings. The defect elimination in the Start/Stop area is especially required, when a closed loop of laser clad track is required which is for instance the case of laser cladding of valve seats [4, 6] or cutting and stumping dies [7].

\section{Experimental}

Laser cladding experiments were carried out on $40 \mathrm{~mm}$ in diameter SS304 steel substrate bar using two different powders: tool steel MicroMelt23 (MM23) and Co based Eutroloy 16012. Chemical compositions of these powders could be found elsewhere [8, 9]. $3 \mathrm{~kW}$ IPG Fiber laser with $600 \mu \mathrm{m}$ optical fiber, 4 axis $\mathrm{CNC}$ table and Metco Twin 10C powder feeder were utilized in laser cladding experiments. Nominal laser power $P$ of $1000 \mathrm{~W}$, laser beam scanning speed $S$ of $5 \mathrm{~mm} / \mathrm{s}$ and powder feeding rates $F$ of 9 and $12 \mathrm{~g} / \mathrm{min}$ were applied during laser cladding of iron and cobalt base coatings, respectively. Defocusing distance of the laser beam focus point from the substrate was $+24 \mathrm{~mm}$, which results in a laser spot size of $3 \mathrm{~mm}$ with the Gaussian distribution of energy density. These processing parameters were selected due to formation of laser tracks with a height of about $1 \mathrm{~mm}$ together with a small amount of dilution with the steel substrate.

Figure 1 show schematically standard laser cladding process controlled by a $\mathrm{CNC}$ program. As individual commands are executed in a sequence, the $\mathrm{CNC}$ command to open laser beam shutter must go before the command for substrate movement. This usually results in small time delay $\tau$ in which the laser beam illuminates the surface that is not in a move. A similar time delay happens at the 


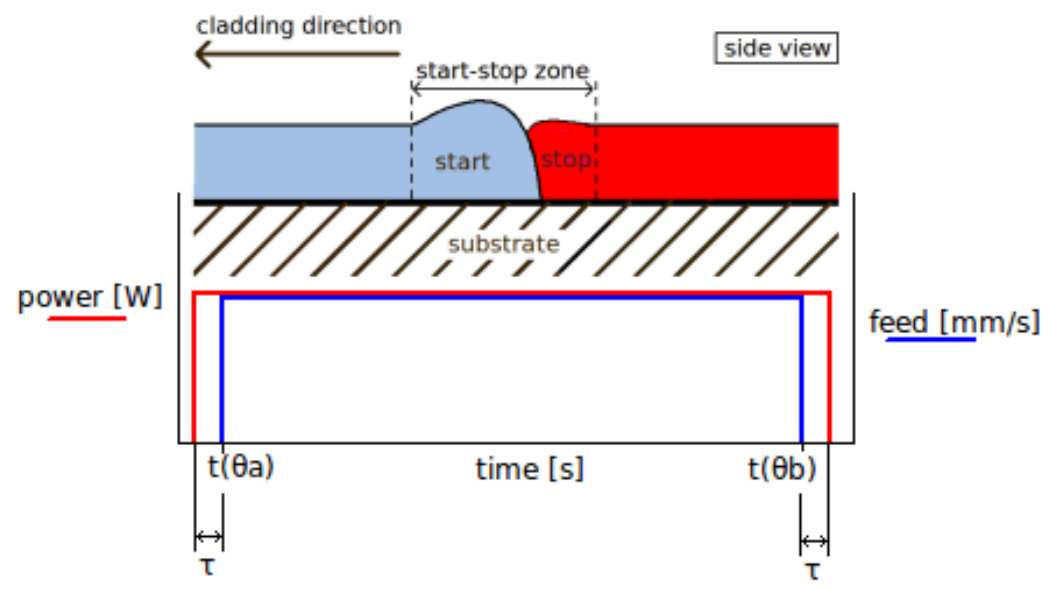

Figure 1: Schematic drawing of standard cladding situation when the laser beam is switched ON shortly before and switched OFF shortly after the substrate movement. The upper part of the diagram shows a longitudinal cross-section of the Start/Stop area of laser track, whereas the lower part demonstrates the laser power and feed rate diagram as a function of time during the whole laser cladding period.

end of the laser cladding process when 'Shutter close' command came after the command to stop the motion. The situation results in a generation of surface defect in the Start/Stop zone.

The modern high power laser systems have a possibility to work in so called "robot mode", when the CNC command does not open the laser shutter directly, but it just initiates an internal laser system program, which allows us to control laser power independently on the $\mathrm{CNC}$ program with some synchronization points, until the "Shutter close" command from the CNC program appears.

\subsection{Experimental laser cladding strategies}

Work in "robot mode" with our laser allows us to try a few different experimental strategies to treat the Start/Stop area more gently. These strategies are graphically summarized in Figures 2a-e. The approach called 'Laser after motion' is the simplest one. It just corrects the standard cladding situation so that the laser beam appears at the substrate after it started to move and also beam is stopped when the substrate is still in a motion. The second approach called in Fig. 2b the 'Gradient power' is a method in which the Start/Stop zone is defined as zone with length of about 5-6 times of laser beam spot size ( 15-18 mm). Inside this zone the laser power is gradually increased at the beginning and gradually decreased at the end of the laser cladding process. 

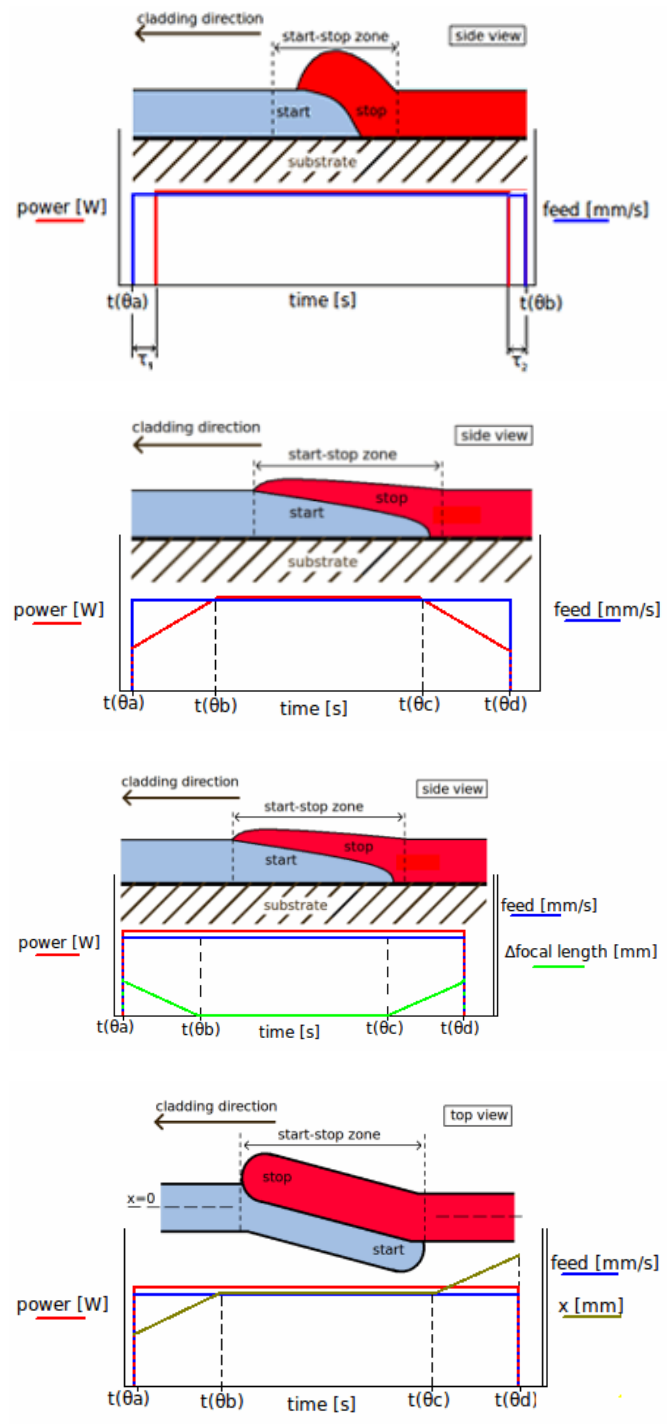

Figure 2d: Track-in/Track-out.

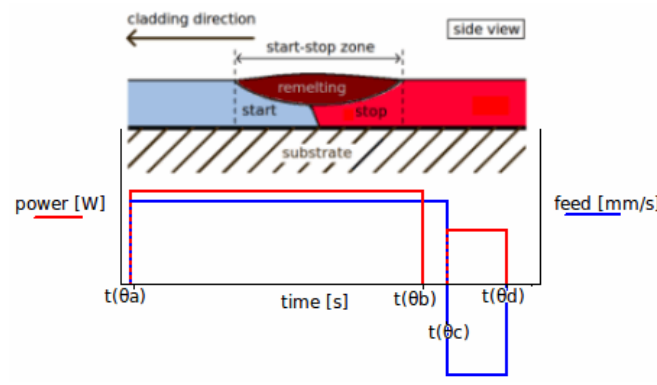

Figure 2a: Laser after motion.

Figure 2b: Gradient power.

Figure 2c: Variable defocus.
Figure 2e: Remelting. 
The next approach shown in Fig. 2c is called 'Variable defocus'. This approach follows a similar idea to the previous one, but the gradual change of the laser power density at the beginning and at the end of laser cladding is realized via changing of laser beam defocus distance while keeping the laser power constant. 'Track-in/Track-out' approach shown in Fig. 2d demonstrates a top view on the laser track. In this method the laser beam gradually steps inside and outside the direction of the laser track within the Start/Stop zone with the 33\% laser tracks side overlapping. The last experimentally tested approach was adopted from the welding community. Here just a simple laser beam remelting inside the Start/Stop zone formed by approach 2a was applied. During the remelting process the laser beam moves with the same speed but in reverse direction, the powder feeder is stopped and only moderate laser power is applied to remelt the laser track only into a particular depth.

\subsection{Experimental methods to study Start/Stop zones}

Standard metallographic methods were used to prepare longitudinal and transversal cross sections of laser tracks produced by different strategies shown in the previous chapter. Light microscope Olympus Vanox-T and field emission gun scanning electron microscope XL30 ESEM equipped with EDS were used to observe the microstructural features and chemical composition inside the Start/Stop zones of laser tracks. CMS Revetest Scratch tester was utilized to measure the profiles of Vickers hardness using the load of $4 \mathrm{~N}$.

\section{Results and discussion}

\subsection{Coating thickness in the Start/Stop zone}

The uniformity of the coating thickness could be one of the measures of the quality of the clad. Abrupt decreases in the laser clad thickness inside the Start/Stop zone are unwanted.

The light optical microscope was used to measure the coating thickness in the vicinity Start/Stop region along longitudinal cross-section of the laser tracks and results for Co-based coatings are summarized in Fig. 3.

It is clear, that a big or small coating thickness decrease is observed in three cases. 'Standard cladding' approach together with 'Laser after motion' shown a substantial drop in coating thickness, while in 'Track-in/Track-out' case shows only a shallow decrease in it. It is important to none, that qualitatively the same results were observed for MM23 laser tracks and that the drop of the coatings thickness happened not only due to the less clad material but also due to non regular shape of the coating/substrate interface, as Fig. 3b demonstrates. The latter case could not be corrected by a final machining of the top surface and therefore it is unacceptable. 

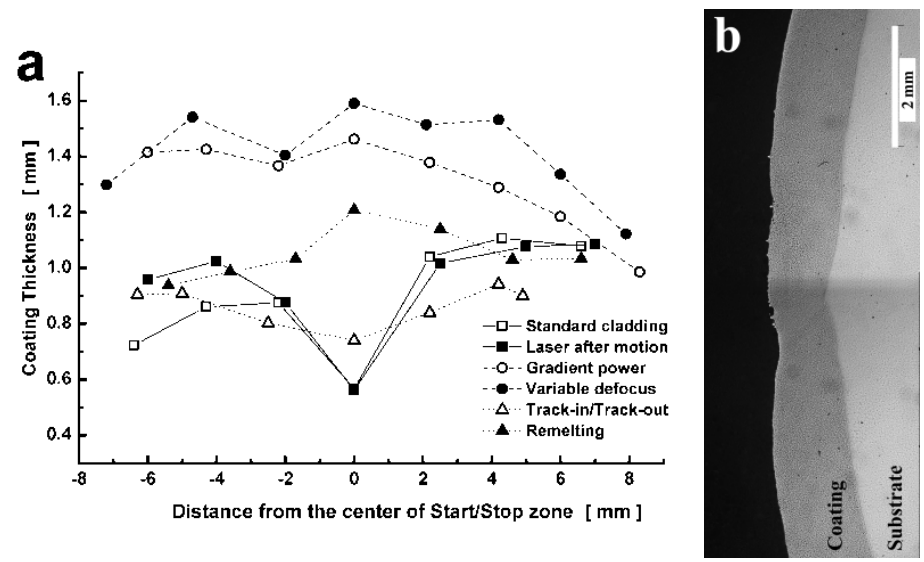

Figure 3: Coating thickness of Co-based coatings in the Start/Stop zone for different experimental approaches (a) and typical coating longitudinal cross section in 'standard cladding' (b).

\subsection{Local hardness near the interface}

Microhardness measurements were made throughout the Start/Stop zone along the longitudinal direction of the clad. Pairs of two indents were positioned in the middle of the coating thickness with a typical horizontal distance between these pairs of about $2 \mathrm{~mm}$. At the centre of the Start/Stop zone three parallel rows of indents were made with a typical vertical distance between them of $0.1 \mathrm{~mm}$. Figure 4 demonstrates a distribution of indents in the vicinity of the Start/Stop zone.

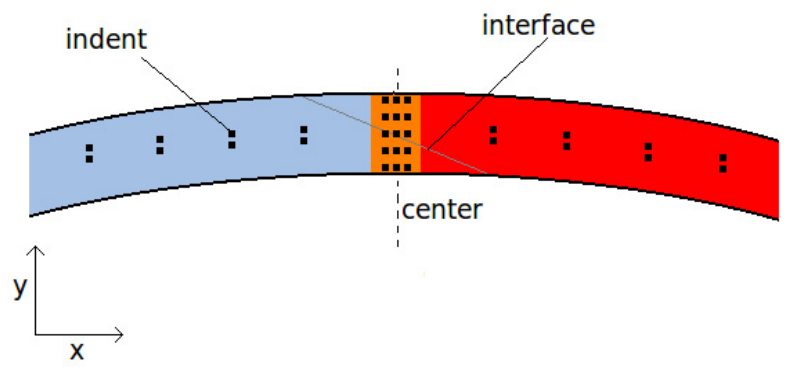

Figure 4: Schematic layout of microhardness indents in Start/Stop zones.

A few typical results of microhardness field in the vicinity of the Start/Stop interface are shown in Tables 1 and 2. Values of hardness of individual indents are shown together with the approximate coordinates of these indents. Moreover, if the interface between start and stop laser tracks is detectable via microstructural features at low magnification, then the position of this interface 
is visualized in these tables by the bold boundary between individual indents. Table 1 demonstrates the 'Standard cladding' situation for a Co-based alloy. In this case the interface between the beginning and the end of the laser track detected via microstructural changes is also accompanied by a sharp change of microhardness. Indeed, such an interface with a sharp change of mechanical properties could be a potential source of local defects during a service of the coating and therefore it should be simply avoided.

Table 1: Vickers microhardness in the vicinity of the Start/Stop area Co-based laser track in 'standard cladding' situation.

\begin{tabular}{|c|c|c|c|c|c|c|c|c|c|}
\hline & & & $\begin{array}{c}\mathrm{y} \\
{[\mathrm{mm}}\end{array}$ & & & & & & \\
\hline & & & 0.7 & & 620 & 561 & & & \\
\hline & & & 0.6 & 601 & 596 & 581 & & & \\
\hline 578 & 522 & 430 & 0.5 & 445 & 620 & 600 & 614 & 590 & 590 \\
\hline 510 & 490 & 430 & 0.4 & 392 & 410 & 593 & 630 & 620 & 551 \\
\hline & & & 0.3 & 381 & 384 & 385 & & & \\
\hline & & & 0.2 & 370 & & 378 & & & \\
\hline & & & 0.1 & 366 & 330 & & & & \\
\hline-6 & -4 & -2 & $\begin{array}{c}\mathrm{x} \\
{[\mathrm{mm}]}\end{array}$ & 0 & 0 & 0 & 2 & 4 & 6 \\
\hline
\end{tabular}

Table 2: Vickers microhardness in the vicinity of the Start/Stop area of MM23 laser track in 'Gradient power' approach.

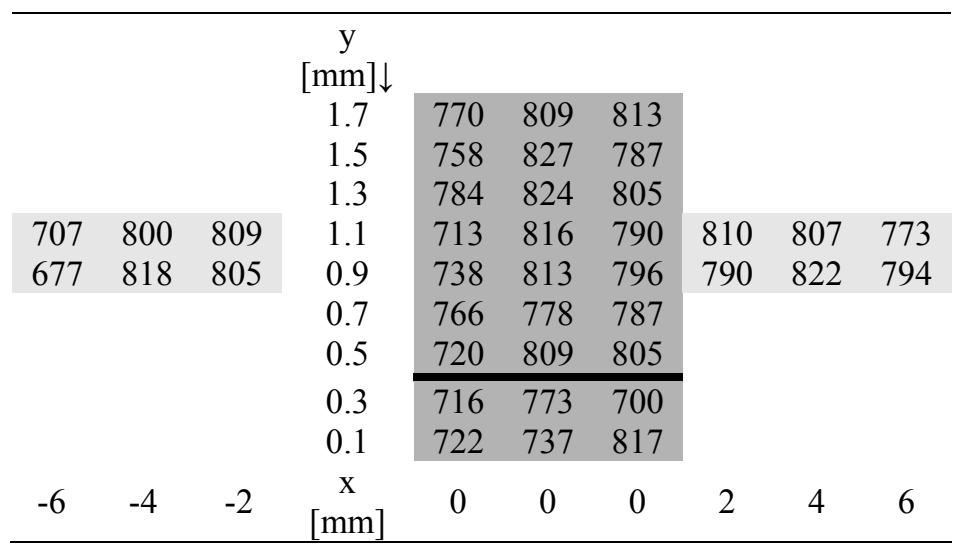

On the other hand, the situation demonstrated in Table 2 does not show any presence of sharp microhardness change and moreover the microhardness values measured close to the interface show the similar values as microhardness values far from this interface. This case is much more favourable for coating in a service than the previous one. 


\subsection{Qualitative evaluation}

In this section we will summarize and evaluate all our experimental approaches to minimize laser cladding defects formed inside the Start/Stop zone. We decided to define four different criteria for this qualitative evaluation.

\section{Change in hardness:}

'+' small change in hardness through the interface $(\Delta \mathrm{HV}<50)$ and the hardness inside the Start/Stop zone is similar to hardness far from this zone;

'-' large change in hardness crossing the interface boundary, hardness in the Start/Stop zone differs from overall hardness of the coating;

\section{Coating thickness uniformity:}

'+' coating thickness does not change substantially in the Start/Stop area;

'-' coating thickness considerably changes;

III. Shape of the coating/substrate interface:

'+' smooth shape of the interface;

'-' uneven shape of the interface;

IV. The visibility of the Start/Stop interface:

'+' Start/Stop interface is not accompanied by a considerable microstructure change;

'-' Microstructure change at this interface is clearly visible in light microscope at a small magnification;

Figure 5 shows two examples of interfaces formed inside the Start/Stop zone. Figure 5a shows and interface between beginning and end of laser track formed by MM23 alloy using 'Gradient power' approach. 'Internal' laser track interface is almost invisible and interface between the clad and substrate is very regular. Figure $5 \mathrm{~b}$ shows the case of 'Track-in/Track-out' method on the same alloy. Internal interface is very well pronounced and Coating/Substrate interface is not regular in the centre of the Start/Stop zone.

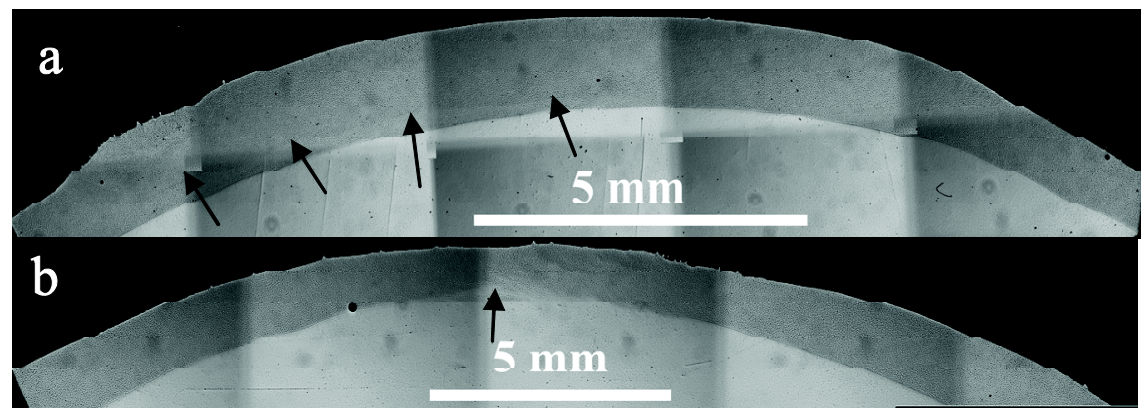

Figure 5: a) Almost invisible Start/Stop interface observed in MM23 laser track treated by 'Gradient power' approach; b) non smooth coating substrate interface present on laser tracks treated by 'Trackin/Track-out' approach. 
The qualitative evaluation of all different experimental methods using these four defined criteria is summarized in Table 3. Evaluation 'o' is attributed to the cases when something between ' + ' and '-' is observed. Considering the strongest requirements for the Start/Stop zone, which means that all four criteria have to be positively fulfilled, only two experimental approaches had satisfied the conditions: 'Gradient power' and 'Variable defocus'. On the other hand, the 'Standard approach' even after a correction for laser beam acting only on the moving substrate: 'Laser after motion' do not fulfil any of the four qualifying conditions.

Table 3: Qualitative evaluation of all experimental methods for both coating materials.

\begin{tabular}{c|cc|cc|cc|cc}
\hline & \multicolumn{2}{|c|}{$\begin{array}{c}\text { I. Hardness } \\
\text { change }\end{array}$} & \multicolumn{2}{|c|}{$\begin{array}{c}\text { II. Uniform } \\
\text { thickness }\end{array}$} & \multicolumn{2}{c|}{$\begin{array}{c}\text { III. Substrate } \\
\text { interface }\end{array}$} & \multicolumn{2}{c}{$\begin{array}{c}\text { IV. Coating } \\
\text { interface }\end{array}$} \\
\hline Exp. approach & MM23 & Co & MM23 & Co & MM23 & Co & MM23 & Co \\
\hline Standard situation & - & - & - & - & - & - & - & - \\
Laser after motion & - & - & - & - & - & - & - & - \\
Gradient power & + & + & + & + & + & + & + & + \\
Variable defocus & + & + & + & + & + & + & + & + \\
Track-in/Track-out & - & - & $\mathbf{0}$ & $\mathbf{0}$ & $\mathbf{0}$ & + & - & $\mathbf{0}$ \\
Remelting & - & + & - & + & $\mathbf{0}$ & $\mathbf{0}$ & + & + \\
\hline
\end{tabular}

\section{Conclusions}

Experimentation with different approaches to eliminate defects in the Start/Stop zone of circular laser tracks from Fe-based and Co-based coating alloys, respectively, have led to the following conclusions:

- In case no special precaution is taken as regards the starting and stopping of the laser track, i.e. using the 'Standard cladding' approach with CNC commands only, the microstructural defects occurred for both coated alloys. Moreover, also the 'Laser after motion' approach incorporates the same defects as the standard cladding. No noticeable difference is found between these two approaches.

- The best approaches to ensure a good clad in the Start/Stop zone for both MicroMelt 23 and Eutroloy 16012 laser coatings are the 'Gradient power' and the 'Variable defocus' set-ups. If the laser power is the parameter that is the easiest to control, than the first approach is preferable. If instead the focal length is easier to control, than the second approach could be applied. Both methods show an increase in height, where the start and end of the laser track overlapped, but this can be machined off. Important is that the coating/substrate interface is smooth and even.

- The remelting approach could also be used for some alloys to reduce the defects in the Start/Stop zone in case when the laser cladding system does not allow a continuous control of laser power or laser defocus parameter. 
- Side overlapping of laser tracks in the Start/Stop zone which is typical for the 'Track-in/Track-out' approach does not fulfill the criterion of a homogeneous hardness value in both tested coating alloys. The 'Trackin/Track-out' approach improves the Start/Stop zone over the standard situation, but is not as good as the three approaches mentioned above.

- The iron based MicroMelt23 alloy is more sensitive to form defects in the Start/Stop zone than the Co based Eutroloy 16012 alloy.

- The uniformity of the hardness inside the Start/Stop zone seems to be the crucial factor that determines defect free cladding in this area.

\section{Acknowledgement}

The work is part of the research program of M2i (Materials Innovation Institute, The Netherlands) Project number: MC7.06259.

\section{References}

[1] de Oliveira U, Ocelik V, De Hosson J. Surface \& coatings technology 2005;197:127-136.

[2] Ocelik V, de Oliveira U, de Boer M, de Hosson J. Surface \& coatings technology 2007;201:5875-5883.

[3] Dahotre N. Advanced Materials \& Processes 2002;160:35-39.

[4] Palumbo G, Pinto S, Tricarico L. Journal of materials processing technology 2004;155:1443-1450.

[5] Kumar S, Goswami GL. Comparative study of wear behaviour of various nibased claddings with stellite- 6 cladding. In: Proceedings of the International Conference on Advances in Surface Treatment: Research and Applications, ASTRA, vol. 2004. 2004. pp. 591-595.

[6] Chang S-S, Wu H-C, Chen C. Mat. \& manufacturing processes 2008;23:708-713.

[7] Hu YP, Chen CW, Mukherjee K. Journal of materials science 1998;33:12871292.

[8] Ocelík V, Bosgra J, de Hosson JTM. Surface and coatings technology 2009;203:3189-3196.

[9] Ocelík V, Furár I, De Hosson JTM. Acta materialia 2010;58:6763-6772. 